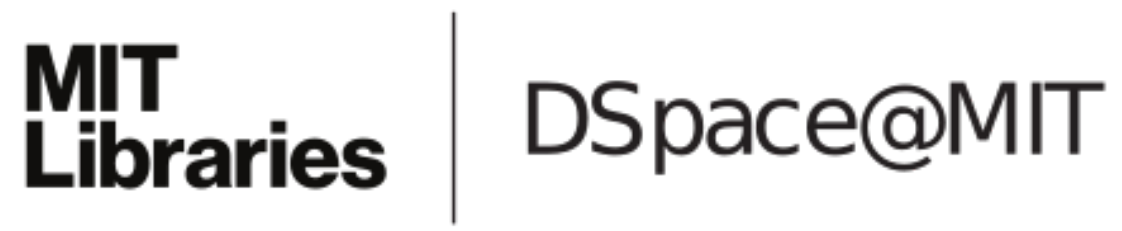

\author{
MIT Open Access Articles
}

\section{Electrowetting-on-Dielectric Actuation of a Vertical Translation and Angular Manipulation Stage}

The MIT Faculty has made this article openly available. Please share how this access benefits you. Your story matters.

Citation: Preston, Daniel J. et al. "Electrowetting-on-Dielectric Actuation of a Vertical

Translation and Angular Manipulation Stage." Applied Physics Letters 109.24 (2016): 244102.

As Published: http://dx.doi.org/10.1063/1.4971777

Publisher: American Institute of Physics (AIP)

Persistent URL: http://hdl.handle.net/1721.1/107712

Version: Author's final manuscript: final author's manuscript post peer review, without publisher's formatting or copy editing

Terms of use: Creative Commons Attribution-Noncommercial-Share Alike 


\title{
Electrowetting-on-Dielectric Actuation of a Vertical Translation and Angular Manipulation Stage
}

\author{
Daniel J. Preston ${ }^{1}$, Ariel Anders ${ }^{\dagger}, 2$, Banafsheh Barabadi ${ }^{\dagger},{ }^{1}$, Evelyn Tio ${ }^{1}$, Yangying Zhu ${ }^{1}$, \\ DingRan Annie Dai ${ }^{1}$, Evelyn N. Wang*, \\ ${ }^{1}$ Department of Mechanical Engineering, Massachusetts Institute of Technology, Cambridge, MA 02139, USA \\ ${ }^{2}$ Department of Electrical Engineering and Computer Science, Massachusetts Institute of Technology, Cambridge, \\ MA 02139, USA \\ ${ }^{\dagger}$ Denotes equal contribution \\ *Corresponding author email: enwang@mit.edu
}

\begin{abstract}
Adhesion and friction during physical contact of solid components in microelectromechanical systems (MEMS) often lead to device failure. Translational stages that are fabricated with traditional silicon MEMS typically face these tribological concerns. This work addresses these concerns by developing a MEMS vertical translation, or focusing, stage that uses electrowetting-on-dielectric (EWOD) as the actuating mechanism. EWOD has the potential to eliminate solid-solid contact by actuating through deformation of liquid droplets placed between the stage and base to achieve stage displacement. Our EWOD stage is capable of linear spatial manipulation with resolution of $10 \mu \mathrm{m}$ over a maximum range of $130 \mu \mathrm{m}$ and angular deflection of approximately $\pm 1^{\circ}$, comparable to piezoelectric actuators. We also developed a model that suggests a higher intrinsic contact angle on the EWOD surface can further improve the translational range, which was validated experimentally by comparing different surface coatings. The capability to operate the stage without solid-solid contact offers potential improvements for applications in micro-optics, actuators, and other MEMS devices.
\end{abstract}

KEYWORDS: Electrowetting on Dielectric (EWOD), Microelectromechanical Systems (MEMS), Tribology, Lab-on-a-Chip 
The efficacy and longevity of actuation-based microelectromechanical systems (MEMS) are limited by tribological issues such as friction and adhesion. ${ }^{1}$ Adhesion typically occurs between small asperities when nominally smooth solid surfaces come into contact, and friction is the result of this adhesion during motion of the surfaces relative to each other. In some cases, the forces from adhesion and friction are comparable to the forces actuating the device, rendering it unusable. In the presence of water vapor, these concerns are amplified; formation of a thin liquid film on solid surfaces and the corresponding capillary and viscous effects that come into play when these surfaces interact lead to stiction, a phenomenon that increases the adhesion between solid asperities and causes small features to stick together due to exceptionally high friction. ${ }^{1-3}$ These reactive forces at the physical contact of solid components lead to wear and removal of material, reducing device lifetime. ${ }^{1,4,5}$ This is of particular importance for traditional MEMS devices fabricated with silicon, a brittle hydrophilic material that is known to have poor tribological properties. ${ }^{6,7}$ It follows that elimination of solid-solid contact during actuation, for example by designing MEMS devices where the actuator plates do not come in to contact, eases tribological and stiction concerns. This work offers a solution for MEMS that eliminates solidsolid contact by using electrowetting to actuate a vertical translation stage.

Electrowetting, a phenomenon whereby the contact angle of a fluid is altered with an applied voltage, allows control of droplet shape. ${ }^{8}$ Recent research has focused on electrowetting on a dielectric, or EWOD, in which an insulating layer is placed between a conductive surface and a droplet which rests on the surface; EWOD can provide much greater droplet deformation than electrowetting on conductive surfaces, and as such is the primary mode of electrowetting used in practical applications. ${ }^{9-12}$ EWOD has played a diverse role in MEMS applications to date, ${ }^{13-17}$ ranging from fluid lenses for optical manipulation ${ }^{18-21}$ to switches for electrical ${ }^{22,23}$ and 
thermal control ${ }^{24,25}$ and thermal management. ${ }^{26}$ EWOD has also been implemented extensively in lab-on-a-chip applications, where arrays of electrodes are activated in sequence to control droplet motion on a surface. ${ }^{27-30}$ With EWOD, controlled droplet vibration and water droplet "jumping,"31 or departure, from hydrophobic surfaces have been demonstrated. ${ }^{31-33}$ EWOD has also been proposed as a method to depin droplets that have been impaled on superhydrophobic surface structures ${ }^{34}$, which is relevant to recent work on superhydrophobicity. ${ }^{35-37}$ However, many opportunities remain to implement EWOD, particularly in the context of MEMS. In this work, we investigated the use of EWOD for its ability to control a MEMS vertical translation, or focusing, stage, thereby providing an alternative to methods which suffer from tribological failure at solid-solid contacts.

EWOD can alter the contact angle of a fluid on a surface and, accordingly, can change the geometry of droplets resting on an EWOD surface when voltage is applied through the droplets. By sandwiching droplets between an electrically conducting stage on one side and an EWOD surface on the other side, a vertical translation stage can be fabricated at the micro-scale as shown schematically in Figure 1a. The stage translates when a voltage is applied across the drops and their contact angle with the EWOD surface decreases. For example, an applied voltage causing a decrease in contact angle on the dielectric layer at the base will result in a broadening and flattening of the droplets and corresponding downward translation of the stage towards the base. This operational mechanism is similar to the capillary force actuator, a class of actuator that relies on deformation of a liquid droplet between two solid surfaces and offers distinct advantages compared to other MEMS actuators. ${ }^{38}$ While the actuation ${ }^{39}$ and dynamics ${ }^{40,41}$ of such devices have been explored theoretically, an experimental device without solid-solid contact has not yet been demonstrated, nor has angular deflection been considered. ${ }^{42}$ 
We developed an axisymmetric iterative numerical model for the four identical droplets in our device, outlined in Figure $1 \mathrm{~b}$ for one of the droplets and later used for comparison with experimental results obtained from a working device, to determine the stage height as a function of the applied voltage. First, the contact angle at the EWOD base was determined with the Lippman-Young equation as a function of the intrinsic contact angle and applied voltage. Then, the droplet curvature in the system was calculated as a function of the internal Laplace pressure, which was determined at the top of the droplet (underside of the stage) by summing one quarter of the stage weight and the surface tension force pulling downwards at the sides of the droplet, $\gamma_{L V} \sin \left(\theta_{t o p}\right)$, and then dividing that quantity by the fixed top contact area. Note that the curvature relies on the initially unknown droplet contact angle at the underside of the stage, $\theta_{\text {top }}$, which is why an iterative solution was implemented. Finally, the complete droplet profile was determined numerically under the constant curvature constraint by iterating until convergence, described in detail in the supplementary information. ${ }^{43}$ The model was used to determine the profiles of droplets under different applied voltages, shown in Figure 1c. The flat region at the top of each profile is the contact with a pinning site on the bottom of the stage, where the constant radius over different applied voltages is consistent with the physical picture. The contact angle at this pinned region varies, as does the radius of the base on the EWOD surface, both of which are expected.

We fabricated the stage by first growing rough copper oxide $(\mathrm{CuO})$ nanoblades on copper foil following a common procedure detailed in the literature ${ }^{35-37}$ and then functionalizing the $\mathrm{CuO}$ with a monolayer of trichloro $(1 \mathrm{H}, 1 \mathrm{H}, 2 \mathrm{H}, 2 \mathrm{H}$-perfluorooctyl)silane (Sigma-Aldrich) to form a superhydrophobic surface. The advancing and receding contact angles of the superhydrophobic $\mathrm{CuO}$ were $\theta_{A}=172^{\circ} \pm 3^{\circ}$ and $\theta_{R}=168^{\circ} \pm 3^{\circ}$, respectively, as measured with a 
microgoniometer (MCA-3, Kyowa). The pinning sites were subsequently formed on the underside of the stage by milling away the $\mathrm{CuO}$ to a negligible depth using an end mill with a diameter of approximately $1.5 \mathrm{~mm}$, thereby exposing the hydrophilic ${ }^{44-46}$ copper and forming a liquid pinning site at the junction of the hydrophilic and superhydrophobic regions to fix the top radius of the droplets. Finally, to establish a non-constraining electrical connection with the stage, a copper wire was soldered in a vertical orientation to a tab at the corner of the stage in order to attach to a sliding attachment mounted above the stage. The total stage mass was 0.080 $\mathrm{g}$, and the load mass (paper with MIT logo shown in Figure 2) was $0.010 \mathrm{~g}$.

Indium-tin-oxide-coated glass slides with resistivity of $10 \Omega / \mathrm{sq}$ were used as the conductive substrate for the EWOD base. The slides were solvent cleaned and plasma cleaned, then coated with a $4 \mu \mathrm{m}$ thick parylene-C layer (VSI Parylene, precision $\pm 1 \mu \mathrm{m}, \theta_{Y} \approx 100^{\circ}$ ) with dielectric strength of $22,000 \mathrm{~V} / \mathrm{m}$ and relative permittivity of $\varepsilon_{r} \approx 3$. Several slides were additionally coated with a sub-micron coating of Teflon aqueous fluoropolymer (AF) as detailed in past work $^{32}$ in order to study the device performance with a higher Young angle at the EWOD base $\left(\theta_{Y} \approx 116^{\circ}\right)$. The water contact angles on the two different EWOD bases at varying applied voltages were characterized by applying voltage through a copper wire electrode inserted into a single $2 \mu \mathrm{L}$ droplet of $0.1 \mathrm{mM} \mathrm{KCl}$ solution in water resting on the EWOD base. The EWOD base was electrically grounded with copper alligator clips penetrating through the dielectric coating to the ITO and subsequently mounted on the stage in front of the high-speed camera. The voltage was varied up to $150 \mathrm{~V}$, and the contact angle was in excellent agreement with the Lippmann-Young prediction (Equation 1) until the saturation voltage for each sample, which occurred at contact angles of $65^{\circ}$ and $74^{\circ}$ for the parylene-C and Teflon AF coatings, respectively. 
The experimental setup consisted of a function generator (AFG 3101, Tektronix) passed through a 400x voltage amplifier (A800, FLC Electronics) with the positive lead wired to the EWOD base and the negative lead attached to the stage via the sliding electrical connection to allow free translation in the vertical z-direction. The stage provided direct electrical connection to the droplets through the conductive hydrophilic copper circles on its underside. The device was both front- and back-lit for high-speed video capture (Phantom v7.1, Vision Research) from 500 to 10,000 frames per second as the experiment was conducted. Four droplets of $0.1 \mathrm{mM}$ $\mathrm{KCl}$ solution in water with a volume of $2 \mu \mathrm{L}$ were carefully pipetted onto the pinning sites on the underside of the stage, which was then inverted and placed onto the EWOD base (the pinned droplets did not fall from the stage). Finally, the stage sliding electrical connection was attached. Figure 2 shows the device with a load on the stage, and the foremost two of the four droplets beneath the stage can be seen.

Images of a typical experiment were captured in Figure 2 (see video in supplementary information ${ }^{43}$ ). At an applied voltage of $150 \mathrm{~V}$, the contact angle decreased from $100^{\circ}$ to $65^{\circ}$ on the parylene-C coated EWOD base and from $116^{\circ}$ to $74^{\circ}$ on the Teflon AF coated EWOD base. This caused the droplets to spread while the volume remained constant and thus resulted in a decrease in stage height as predicted by the model. Tests at intermediate voltages in Figure $3 \mathrm{c}$ show good agreement with the model prediction, which is a combination of the Lippman-Young equation shown in Figure 3a (including saturation) and the stage height as a function of base contact angle for a $2 \mu \mathrm{L}$ droplet generated by the iterative solution (plotted in supplementary material $\left.^{43}\right)$.

To eliminate solid-solid contact and the accompanying stiction and tribological concerns, the stage was reconfigured to remove the requirement for the sliding electrical connection. This 
was achieved by separating the Teflon AF coated EWOD base into two electrically insulated components, each holding two droplets (Figure 4a). Then, voltage was applied from one insulated section of the EWOD base to the other, forming a circuit comprised of two capacitors (the dielectric regions at the base of the droplets on each of the insulated EWOD base sections). Since each of these series capacitors carry half of the applied voltage, twice the voltage required in the previous configuration is required for the same stage deflection. The stage deflection in this configuration was experimentally demonstrated to be equivalent to the previous (wired) configuration and in good agreement with the model, as shown in Figure 4b.

Additionally, the stage can provide angular deflection. The configuration was further modified to keep the electrically separated EWOD base but once again include the stage sliding electrical connection, which was grounded (Figure 4c). When a voltage is applied to either insulated section of the EWOD base, that side of the stage is displaced downwards while the other side remains unperturbed, resulting in angular deflection. To test this configuration, we constructed a varying voltage that first actuated one side of the stage, and then actuated the other side of the stage. The function generator/amplifier output was set to increase from $0 \mathrm{~V}$ to $150 \mathrm{~V}$ and then decrease back to $0 \mathrm{~V}$ repeatedly as a sine wave with amplitude $75 \mathrm{~V}$, offset $+75 \mathrm{~V}$, and period 2 sec. This signal was followed by a microcontroller (UNO R3, Arduino) which used a motor shield (L298P, Arduino) to switch relays (7266K64, McMaster-Carr) that alternated the applied voltage between the two sides of the EWOD base each time the signal bottomed out at 0 $\mathrm{V}$, leaving the non-active side of the EWOD base at $0 \mathrm{~V}$. The result of the signal applied to this configuration is shown in Figure 4d, where each side of the stage deflected by approximately 130 $\mu \mathrm{m}$ when the voltage was applied (diamond and square symbols), in agreement with the uniform 
vertical stage displacement demonstrated previously, and the stage angular displacement varied from approximately $-1^{\circ}$ to $+1^{\circ}$ (hollow circular symbols).

Combining the two modified configurations above could yield an angular deflection stage that does not require any solid-solid contact (no stage electrode connection). This is possible by separating the EWOD base into four electrically isolated sections, one for each droplet, and then essentially controlling the deflection of two adjacent droplets by applying a voltage across the EWOD base beneath those droplets. Such a configuration would also allow angular deflection along any axis of rotation within the plane of the stage. A further expansion of this concept could build on past work in which the EWOD surface was separated into an array of isolated electrodes which were actuated separately such that lateral droplet motion was induced. ${ }^{47-50}$ Operation of the stage described in the present work on such an array of electrodes could allow for lateral as well as vertical translation.

This work shows a MEMS vertical translation stage that uses EWOD as the actuating mechanism. The EWOD stage was capable of linear spatial manipulation with resolution of 10 $\mu \mathrm{m}$ over a maximum range of $130 \mu \mathrm{m}$, which can be readily improved and tailored to specific applications in future device generations with guidance from the validated model developed in the present work. Specifically, both model and experiment show that a higher intrinsic contact angle on the EWOD base improves absolute range, and reduction of contact angle hysteresis, ${ }^{51}$ possibly by addition of a lubricant to the surface ${ }^{52-54}$ or careful control of contaminants, ${ }^{45}$ will increase resolution. In addition, angular deflection of approximately $\pm 1^{\circ}$ was demonstrated, and the maximum range and angular deflection are comparable to another MEMS alternative, piezoelectric actuators. The capability to operate the stage without any solid-solid contact makes 
this a desirable potential solution to stiction and tribology concerns for improvement of applications in micro-optics, actuators, and other MEMS. 


\section{Acknowledgements}

We thank Professors John W. M. Bush and Michael Cima at MIT for fruitful discussions regarding the work. We gratefully acknowledge funding support from the Office of Naval Research (ONR) with Dr. Mark Spector as program manager. D. J. Preston acknowledges funding received from the National Science Foundation (NSF) Graduate Research Fellowship under Grant No. 1122374. We also acknowledge the support from the NSF through the Major Research Instrumentation Grant for Rapid Response Research (MRI-RAPID). 


\section{References}

1 Sujeet K. Sinha, N. Satyanarayana, and Seh Chun Lim, Nano-tribology and materials in MEMS. (Springer Berlin Heidelberg, 2013), pp.1 online resource (viii.

2 C. H. Mastrangelo, Materials Science of Microelectromechanical Systems (Mems) Devices Ii 605, 105 (2000).

3 B. Bhushan, J Vac Sci Technol B 21 (6), 2262 (2003).

4 Daniel J. Fonseca and Miguel Sequera, Journal of Quality and Reliability Engineering 10 (1155), 820243 (2011).

$5 \quad$ J. A. Williams and H. R. Le, J Phys D Appl Phys 39 (12), R201 (2006).

$6 \quad$ X. Liu, K. Kim, and Y. Sun, J Micromech Microeng 17 (9), 1796 (2007).

7 C. Ataman, Y. Petremand, W. Noell, H. Urey, M. Epitaux, and N. F. De Rooij, MEMS, MOEMS, and Micromachining II 6186, 18603 (2006).

$8 \quad$ M. G. Lippmann, Ann. Chim. Phys. 5 (1875).

9 N. Verplanck, Y. Coffinier, V. Thomy, and R. Boukherroub, Nanoscale Res Lett 2 (12), 577 (2007).

10 M. J. Schertzer, S. I. Gubarenko, R. Ben-Mrad, and P. E. Sullivan, Langmuir 26 (24), 19230 (2010).

11 M. J. Schertzer, S. I. Gubarenko, R. Ben-Mrad, and P. E. Sullivan, International Mechanical Engineering Congress and Exposition - 2012, Vol 9, Pts a and B, 61 (2013).

12 C. T. Burkhart, P. D. Dunning, and M. J. Schertzer, Proceedings of the Asme 13th International Conference on Nanochannels, Microchannels, and Minichannels, 2015 (2015).

13 N. Y. Kim, S. M. Hong, and S. S. Park, International Mems Conference 2006 34, 650 (2006).

14 Y. Mita, Y. Li, M. Kubota, S. Morishita, W. Parkes, L. I. Haworth, B. W. Flynn, J. G. Terry, T. B. Tang, A. D. Ruthven, S. Smith, and A. J. Walton, Solid State Electron 53 (7), 798 (2009).

15 I. Moon and J. Kim, Sensor Actuat a-Phys 130, 537 (2006).

16 S. K. Fan, C. Hashi, and C. J. Kim, Mems-03: Ieee the Sixteenth Annual International Conference on Micro Electro Mechanical Systems, 694 (2003). 
17 J. Berthier, P. Dubois, P. Clementz, P. Claustre, C. Peponnet, and Y. Fouillet, Sensor Actuat aPhys 134 (2), 471 (2007).

18 T. Krupenkin, S. Yang, and P. Mach, Appl Phys Lett 82 (3), 316 (2003).

19 X. F. Zeng and H. R. Jiang, J Phys D Appl Phys 46 (32) (2013).

20 F. Krogmann, W. Monch, and H. Zappe, Journal of Optics A - Pure and Applied Optics 8 (7), S330 (2006).

21 M. A. Bucaro, P. R. Kolodner, J. A. Taylor, A. Sidorenko, J. Aizenberg, and T. N. Krupenkin, Langmuir 25 (6), 3876 (2009).

22 P. Sen and C. J. Kim, J Microelectromech S 18 (1), 174 (2009).

23 P. Sen and C. J. Kim, Ieee 22nd International Conference on Micro Electro Mechanical Systems (Mems 2009), 904 (2009).

24 J. Gong, G. Cha, Y. S. Ju, and C. J. Kim, Mems 2008: 21 st Ieee International Conference on Micro Electro Mechanical Systems, Technical Digest, 848 (2008).

25 A. R. McLanahan, C. D. Richards, and R. F. Richards, J Micromech Microeng 21 (10) (2011).

26 J. T. Cheng and C. L. Chen, Exp Fluids 49 (6), 1349 (2010).

$27 \quad$ S. Haeberle and R. Zengerle, Lab Chip 7 (9), 1094 (2007).

28 J. Gong and C. J. Kim, Lab Chip 8 (6), 898 (2008).

29 G. J. Shah, A. T. Ohta, E. P. Y. Chiou, M. C. Wu, and C. J. Kim, Lab Chip 9 (12), 1732 (2009).

30 S. K. Cho, H. J. Moon, and C. J. Kim, J Microelectromech S 12 (1), 70 (2003).

31 S. J. Lee, S. Lee, and K. H. Kang, Appl Phys Lett 100 (8) (2012).

32 A. Cavalli, D. J. Preston, E. Tio, D. W. Martin, N. Miljkovic, E. N. Wang, F. Blanchette, and J. W. M. Bush, Phys Fluids 28 (2) (2016).

33 W. C. Nelson and C. J. Kim, Journal of Adhesion Science and Technology 26 (12-17), 1747 (2012).

34 F. Lapierre, Y. Coffinier, R. Boukherroub, and V. Thomy, Langmuir 29 (44), 13346 (2013). 
N. Miljkovic, D. J. Preston, R. Enright, and E. N. Wang, Nat Commun 4 (2517) (2013).

N. Miljkovic, D. J. Preston, R. Enright, and E. N. Wang, Acs Nano 7 (12), 11043 (2013). Conference on Micro Electro Mechanical Systems (Mems) (2012).

43 See supplementary material at (link) for a video of the stage in operation; experiment schematic; derivation of the Lippmann-Young equation; consideration of stage-base electrostatic attraction; and pseudocode describing the numerical model solution.

$44 \quad$ M. E. Schrader, J Phys Chem-Us 78 (1), 87 (1974).

45 D. J. Preston, N. Miljkovic, J. Sack, R. Enright, J. Queeney, and E. N. Wang, Appl Phys Lett 105 (11601) (2014).

46 D. J. Preston, D. L. Mafra, N. Miljkovic, J. Kong, and E. N. Wang, Nano Lett 15 (5), 2902 (2015).

47 J. K. Park, S. J. Lee, and K. H. Kang, Icnmm 2009, Pts a-B, 143 (2009).

48 Q. Ni, D. E. Capecci, and N. B. Crane, Sensor Actuat a-Phys 247, 579 (2016).

49 H. L. Ricks-Laskoski, M. A. Buckley, and A. W. Snow, J Appl Polym Sci 110 (6), 3865 (2008).

50 C. G. Cooney, C. Y. Chen, M. R. Emerling, A. Nadim, and J. D. Sterling, Microfluid Nanofluid 2 (5), 435 (2006).

$51 \quad$ L. C. Gao and T. J. McCarthy, Langmuir 25 (24), 14105 (2009).

52

H. J. J. Verheijen and M. W. J. Prins, Langmuir 15 (20), 6616 (1999). 
53 T. S. Wong, S. H. Kang, S. K. Y. Tang, E. J. Smythe, B. D. Hatton, A. Grinthal, and J. Aizenberg, Nature 477 (7365), 443 (2011).

$54 \quad$ A. Lafuma and D. Quere, Epl-Europhys Lett 96 (5) (2011). 
a)
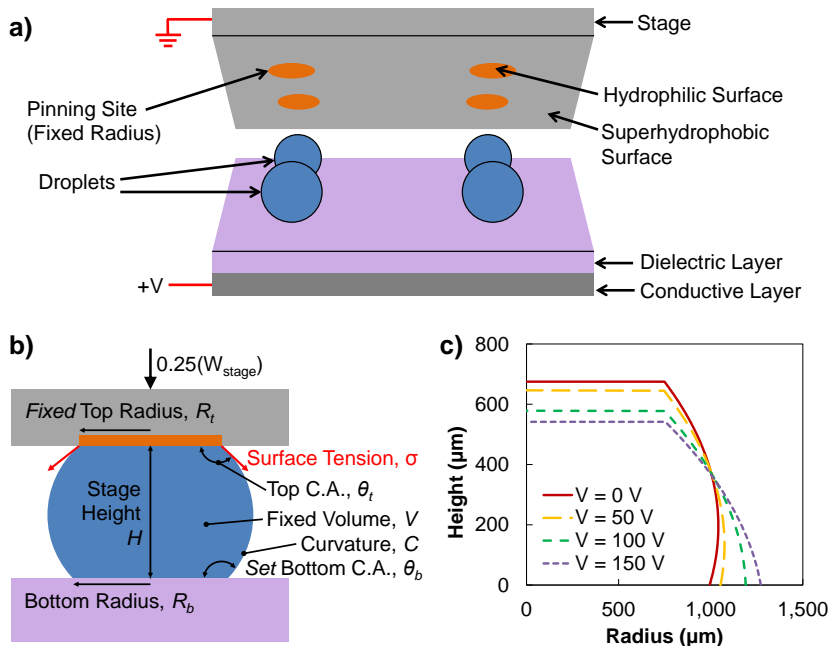

Figure 1. (Single Column, $\mathbf{A R}=\mathbf{1 . 3 5}$ ) (a) The electrowetting-actuated stage is shown schematically. The tops of the water droplets contact the underside of the stage at electrically conducting hydrophilic copper pinning sites surrounded by a superhydrophobic surface, and the bottoms of the droplets rest on the insulated electrowetting on dielectric base. (b) The device actuation can be modeled through a droplet of fixed volume, $V$, where the droplet-stage interface has fixed radius but variable contact angle at the pinned radius, $R_{t}$, between the hydrophilic and superhydrophobic regions on the stage underside, and the droplet-base interface has a contact angle, $\theta_{b}$, set by the applied voltage using the Lippmann-Young equation but a variable radius.

(c) The expected stage height can be determined by the axisymmetric model, as shown in the droplet profiles as a function of applied voltage for a $2 \mu \mathrm{L}$ droplet on a surface with a Young contact angle of $110^{\circ}$. The flat regions at the top of the droplet profiles indicate contact with the region of fixed radius on the stage. 


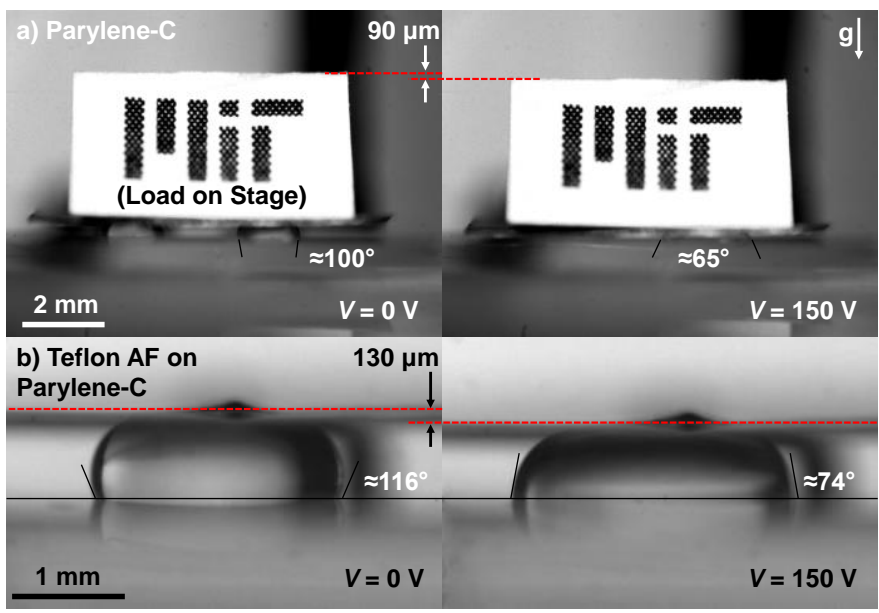

Figure 2. (Single Column, $\mathbf{A R}=\mathbf{1 . 4 3})$ Experimental images of the initial neutral state $(0 \mathrm{~V})$ and the stage vertical translation at an applied voltage of $150 \mathrm{~V}$, which resulted in a stage deflection of (a) $90 \mu \mathrm{m}$ with the bare parylene-C surface and (b) $130 \mu \mathrm{m}$ with the Teflon AF-coated surface (zoomed in to one droplet in this case) compared to the initial position. The images at $150 \mathrm{~V}$ represent the maximum stage translation for each surface using the $2 \mu \mathrm{L}$ droplets because the electrowetting effect reached saturation and an increase in voltage did not result in further stage deflection. 

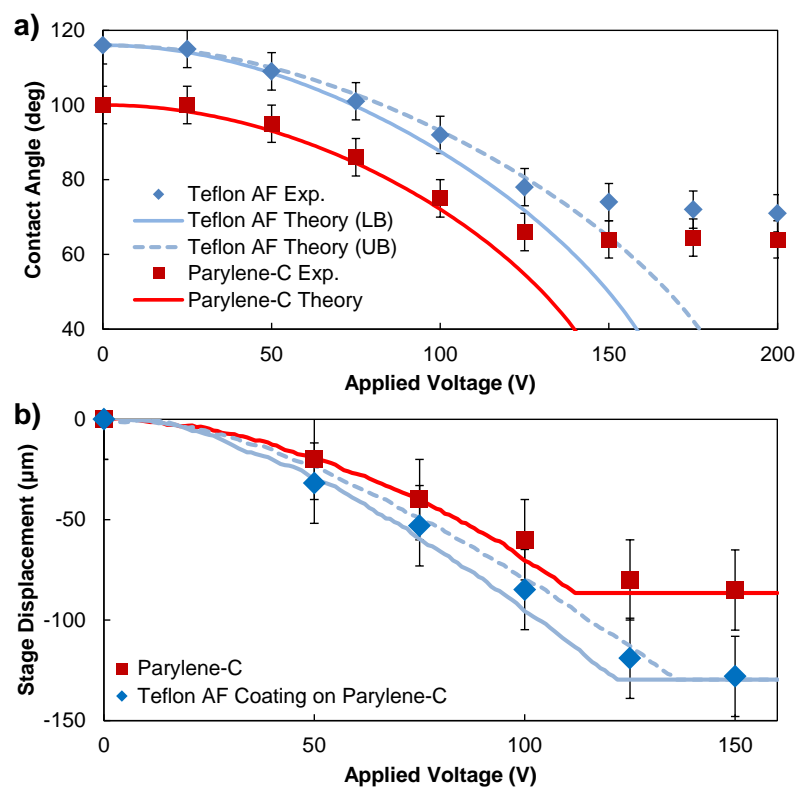

Figure 3. (Single Column, $\mathbf{A R}=\mathbf{1 . 0 1}$ ) (a) Experimental measurements for a single droplet on both parylene-C (red) and Teflon AF-coated (blue) surfaces as a function of applied voltage, with the corresponding Lipmann-Young theory shown as solid lines (for Teflon AF-coated surface, the dashed line represents the upper bound (UB) and the solid line the lower bound (LB) in dielectric layer thickness due to uncertainty arising from the coating procedure). The droplet contact angle behavior agrees with the Lipmann-Young theory until saturation. (b) When the Lippman-Young equation is combined with the numerical droplet profile solution for stage displacement as a function of bottom surface contact angle, the model can predict the stage height as a function of applied voltage, which is in good agreement with the experimental results for stage deflection. 

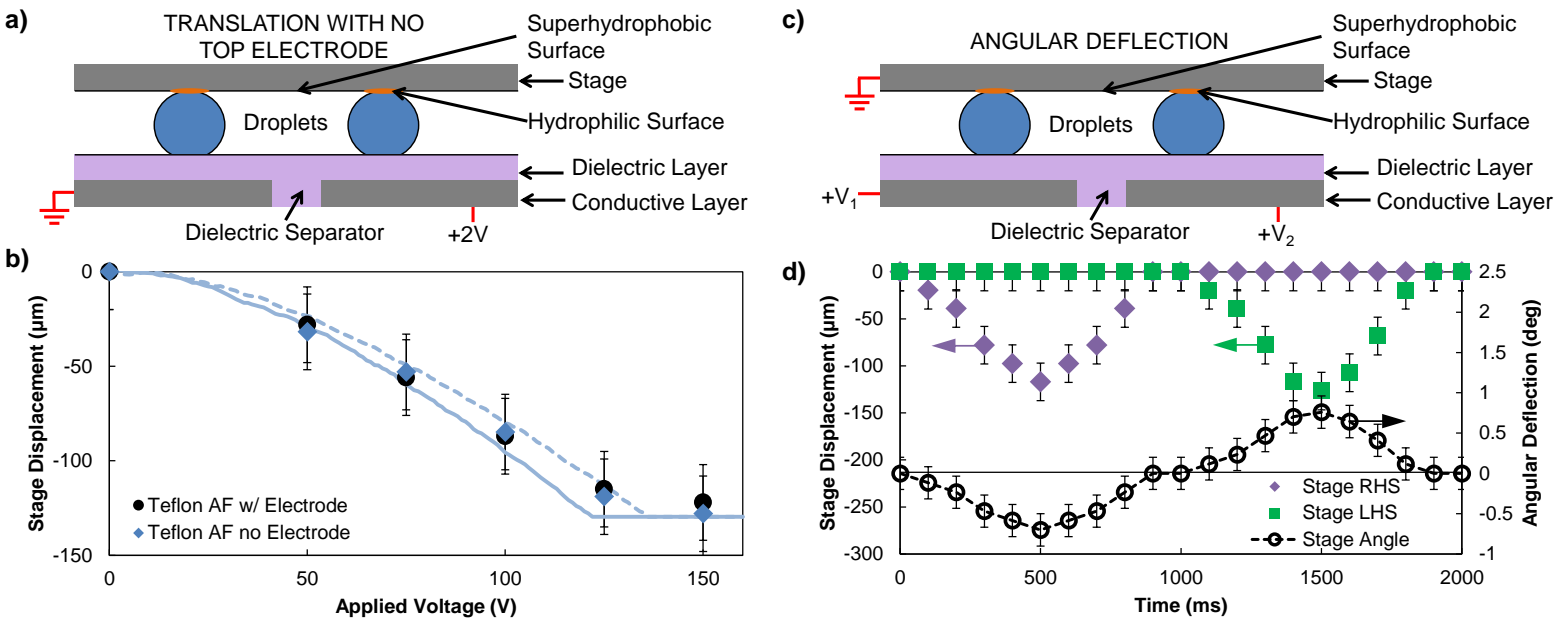

Figure 4. (Double Column, $A R=2.43$ ) The electrical connections of the device can be configured to eliminate the top surface sliding electrode and to induce angular deflection. The elimination of the top electrode, achieved by applying the voltage to two droplets in series from a base with a dielectric separator between two pairs of droplets (a), provides the same stage deflection as a function of voltage as the wired version (b). Keeping the base separated by a dielectric barrier at the center allows for angular deflection of the stage when the top of the stage is grounded by applying differing voltages to the two sections of the base (c), which reached approximately one degree in the current configuration (d). These two approaches can be combined for angular deflection without a direct electrical connection to the stage by dividing the base into four electrically insulated regions, one to control each droplet. 


\section{Supplementary Material}

\section{Supplementary Video S1}

This video shows vertical deflection of the stage as a square wave with a peak-to-peak amplitude of $150 \mathrm{~V}$ and an offset of $75 \mathrm{~V}$ (applied voltage alternating between 0 and $+150 \mathrm{~V}$ ) and a frequency of $50 \mathrm{~Hz}$ is applied to the device. The video has been slowed 100x and illustrates both spatial and temporal control of the stage height.

\section{Experimental Setup}

The experimental setup is shown schematically here:

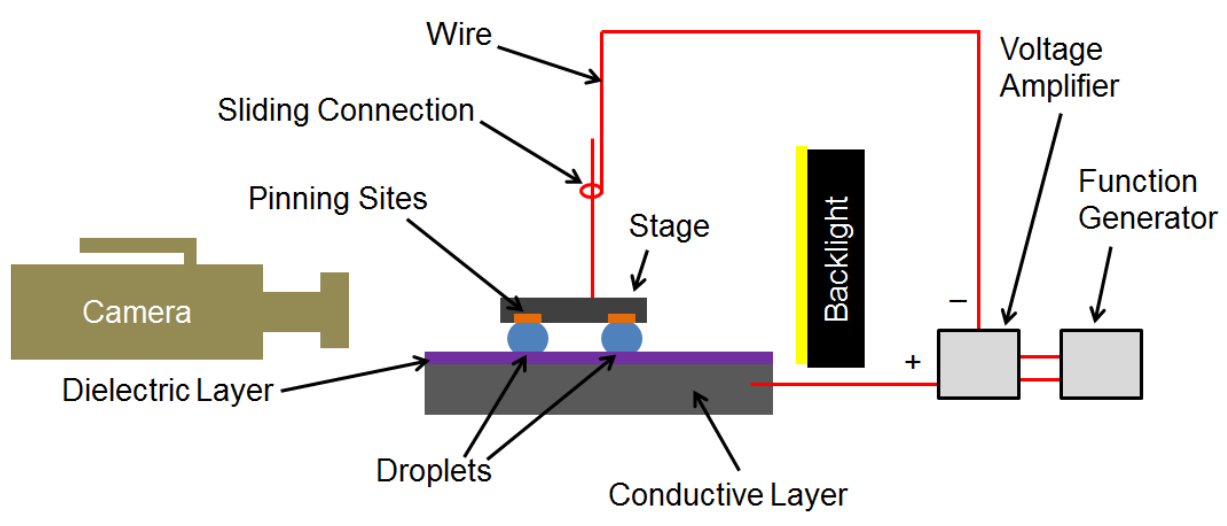

Figure S1. Schematic of the experimental setup used to characterize the performance of the EWOD z-stage both statically and dynamically. Images were captured from the side of the device to enable quantification of droplet contact angle and stage height as the voltage was applied to the device and the drops deformed.

\section{Derivation of the Lippmann-Young Equation}

For a liquid at rest on a flat and chemically homogeneous solid surface, the differential energy required to advance the three-phase contact line (i.e., where the fluid, solid, and surrounding air or vapor meet) by a differential distance is:

$$
d E=\left(\sigma_{S V}-\sigma_{S L}-\sigma_{L V} \cos \left(\theta_{Y}\right)\right) d x
$$

where $\sigma_{S V}$ and $\sigma_{S L}$ are the surface energies of the solid/vapor and solid/liquid interfaces, respectively, $\sigma_{L V}$ is the surface tension of the liquid/vapor interface, and $\theta_{Y}$ is the equilibrium contact angle. The equilibrium contact angle between these three phases, measured within the liquid, is determined by taking $d E / d x=0$. The result, first defined in 1805 by Young ${ }^{1}$, is: 


$$
\cos \left(\theta_{Y}\right)=\frac{\sigma_{S V}-\sigma_{S L}}{\sigma_{L V}}
$$

Equation S2 is central to wetting science, and electrowetting is not an exception. A typical EWOD experimental setup consists of a conductive substrate covered by a thin dielectric layer of thickness $t$ as the flat solid surface. An electrode is placed into the liquid phase, and a voltage is applied across the electrode and the conductive substrate. This applied voltage results in a capacitive storage of energy across the thin dielectric layer as charge separates within the liquid. ${ }^{2}$ The energy stored in a flat plate capacitor is:

$$
E_{c}=\frac{1}{2} \frac{\varepsilon_{r} \varepsilon_{0} A}{t} V^{2}
$$

where $\varepsilon_{r}$ and $\varepsilon_{0}$ are the relative and vacuum permittivities, respectively, $A$ is the area of the capacitor plate, $t$ is the distance between plates, and $V$ is the applied voltage. Reformulating Equation S3 on a per-unit-area basis to match the dimensions of the other surface energies involved and following the differential energy method used previously, the relationship:

$$
\cos \left(\theta_{L Y}\right)=\frac{1}{\sigma_{L V}}\left(\sigma_{S V}-\sigma_{S L}+\frac{\varepsilon_{r} \varepsilon_{0} V^{2}}{2 t}\right)
$$

for the Lippmann-Young contact angle, $\theta_{L Y}$, is obtained, which can be rearranged in terms of the equilibrium contact angle:

$$
\cos \left(\theta_{L Y}\right)=\cos \left(\theta_{Y}\right)+\frac{\varepsilon_{r} \varepsilon_{0} V^{2}}{2 \sigma_{L V} t}
$$

Note that the dimensionless electrowetting parameter in this equation can only be positive; therefore, an applied voltage can only serve to decrease the Lippmann-Young contact angle relative to the equilibrium contact angle.

An important aspect of wetting not mentioned to this point is the effect of roughness and chemical inhomogeneity on a surface. These deviations from an ideal surface are local energy barriers that cause a difference between the contact angle observed during advancing of the three-phase contact line over the surface and receding of the contact line. The difference in these angles is termed the contact angle hysteresis, and it can result in pinning of droplets to which a voltage has been applied (i.e., the droplets do not necessarily return to their original shape upon release of the voltage). This is a concern for design of the EWOD base - the contact hysteresis must be as low as possible. 


\section{Electrostatic Attraction between Stage and EWOD Base}

The effect of electrostatic interaction between the stage and EWOD base was considered by applying the scaling shown in Equation S6 for the ratio of electrostatic force between two charged plates to gravitational force (weight) of the stage:

$$
\frac{F_{E}}{F_{g}}=\frac{\frac{\varepsilon_{0} A V^{2}}{2 d^{2}}}{m g}=\frac{4 \times 10^{-5} N}{8 \times 10^{-4} N} \ll 1
$$

where the stage area $A$ was approximately $1 \mathrm{~cm}$ x $1 \mathrm{~cm}$, the voltage was taken to be the maximum used in the present work of $150 \mathrm{~V}$, and the spacing $d$ was taken as the closest spacing between the stage and the EWOD substrate observed in the present work of $\approx 500 \mu \mathrm{m}$ (note that the permittivity of water was not taken into account due to the relatively small total area between the plates occupied by water compared to air). Since the electrostatic force was much less than the weight of the stage itself, the electrostatic force was neglected in the model. This is a conservative estimate considering that the stage load and the downward force from the water contact line on the underside of the stage were not considered in this analysis.

\section{Model Description and Pseudocode}

We developed a model for one of the four identical droplets that comprised the stage actuation mechanism to determine the stage height as a function of the applied voltage. First, the contact angle at the EWOD base was determined. For a liquid at rest on a flat and chemically homogeneous solid surface, the differential energy required to advance the three-phase contact line can be used to determine the equilibrium contact angle between these three phases by taking $d E / d x=0$. The result, often referred to as the Young angle, is altered during EWOD by applying a voltage through the fluid and across a thin dielectric layer of thickness $t$ which coats the solid surface. This dielectric layer stores capacitive energy and effectively alters the liquid-solid interface net surface energy, resulting in the Lippman-Young equation:

$$
\cos \left(\theta_{L Y}\right)=\cos \left(\theta_{Y}\right)+\frac{\varepsilon_{r} \varepsilon_{0} V^{2}}{2 \sigma_{L V} t}
$$

where $\theta_{L Y}$ is the contact angle with an applied voltage, $\theta_{Y}$, is the Young angle, $\varepsilon_{r}$ and $\varepsilon_{0}$ are the relative and vacuum permittivities, respectively, and $V$ is the applied voltage. Note that the dimensionless electrowetting parameter in this equation can only be positive; therefore, an

applied voltage can only serve to decrease the Lippmann-Young contact angle relative to the Young angle. 
As shown in Equation S7, the relationship between the applied voltage and the droplet contact angle on the EWOD base is found from the Lippmann-Young equation. Therefore, the majority of the modeling effort focused on determining the droplet shape under the constraints detailed in Figure $1 \mathrm{~b}$ and Figure S2, namely, fixed volume, fixed radius but variable contact angle at the underside of the stage, and variable radius but prescribed contact angle at the EWOD base. An axisymmetric iterative numerical solution was developed for the four identical droplets. The droplet curvature in the system is a function of the internal Laplace pressure, which is determined at the top of the droplet (underside of the stage) by summing one quarter of the stage weight and the surface tension force pulling downwards at the sides of the droplet, $\gamma_{L V} \sin \left(\theta_{t o p}\right)$, and then dividing by the fixed top contact area. The electrostatic attraction between the oppositely charged stage and EWOD base was neglected according to Equation S6. Note that $\theta_{\text {top }}$ is initially unknown and needs to be solved for; it is initialized to $90^{\circ}$. Each iteration of the model starts at the prescribed base contact angle and a given value for the base radius and increments up the sidewall of the droplet by steps of size $d s, d s \rightarrow 0$, updating the wall slope at each step according to the local radius and the surface curvature, continuing until the expected volume is reached. At this point (which must correspond to contacting the base of the stage), the radius is compared to the expected radius of the pinning site on the underside of the stage, and if the values are not equal, the base radius is incremented by a small amount and the iteration is run again. Once the model-predicted top radius is equivalent to the pinned radius at the underside of the stage, the model-predicted contact angle at the underside of the stage is then compared to $\theta_{t o p}$ used to set the curvature, and $\theta_{t o p}$ and the curvature are adjusted accordingly and the entire process repeated. This process continues until the model result converges to within $0.1^{\circ}$ of the initial value of $\theta_{t o p}$ while also matching the pinned top radius and the fixed volume constraint, having started from the prescribed base contact angle. This nested loop structure solves for a droplet profile using a step size $d s=2 \mu \mathrm{m}$, with no change in solution at smaller step sizes.

The model pseudocode is shown here for use in future design and improvement of the stage. As an interesting side note, the model yielded a catenoid when the Laplace pressure was set to zero, which served to validate the model. The accompanying schematic in Figure S2 is provided for reference. Additionally, the model results for the intrinsic contact angles of the two surfaces used in experimental device demonstration in the manuscript are plotted in Figure S3 below; the stage displacement as a function of the base contact angle shown here is used in combination with the base contact angle as a function of the applied voltage (Figure 3(a), Lippmann-Young equation) in order to determine the model prediction of stage displacement (Figure 3(b)). 


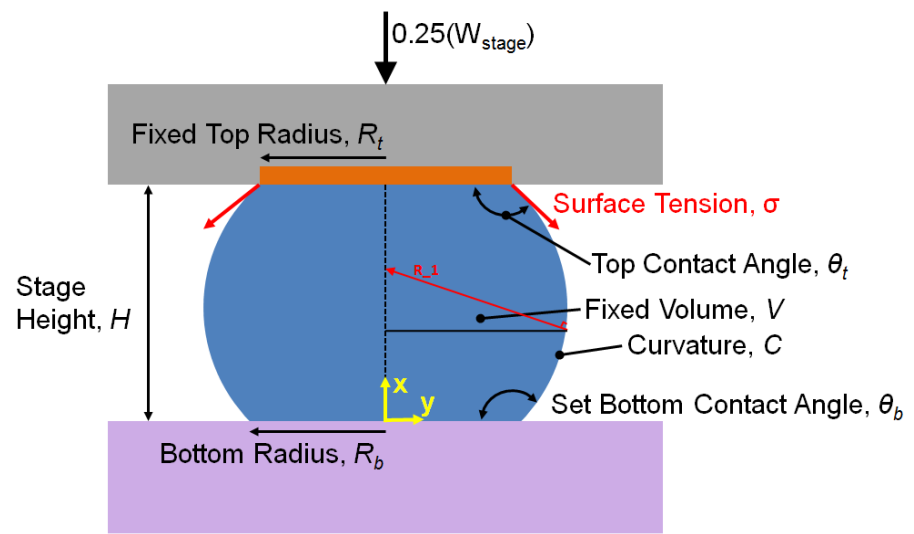

Figure S2. Schematic to accompany pseudocode. The device actuation can be modeled through a droplet of fixed volume, $V$, where the droplet-stage interface has fixed radius but variable contact angle at the pinned radius, $R_{t}$, between the hydrophilic and superhydrophobic regions on the stage underside, and the droplet-base interface has a contact angle, $\theta_{b}$, set by the applied voltage using the Lippmann-Young equation but a variable radius. The coordinate system is as shown, with "y" representing a radial coordinate in the axisymmetric system.

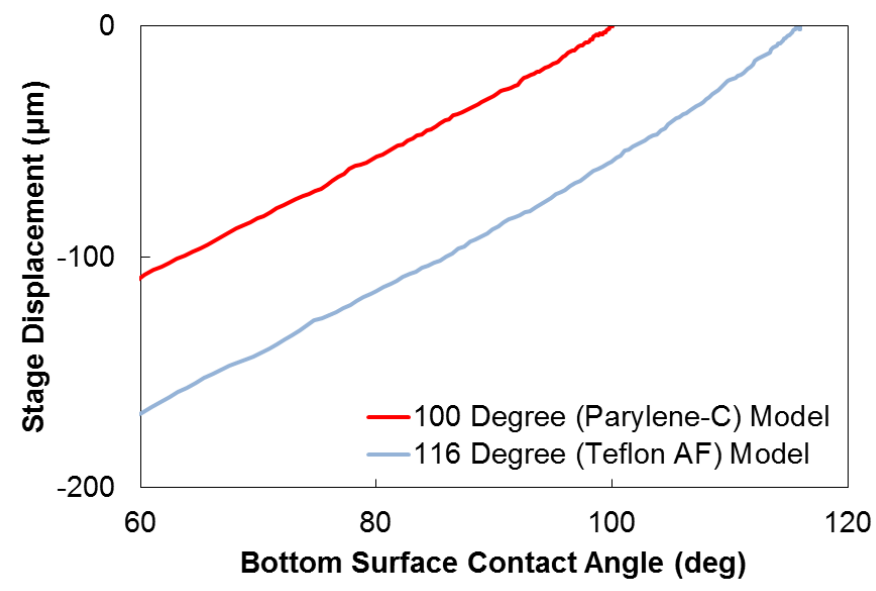

Figure S3. Model results for the two different surfaces experimentally tested in the manuscript. The stage displacement is plotted as a function of the bottom surface contact angle.

\section{Pseudocode:}

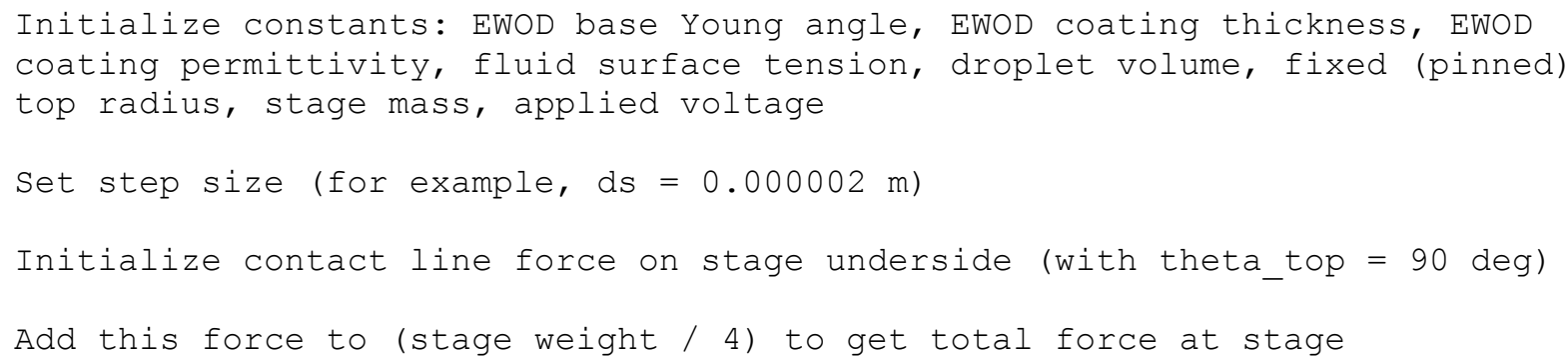


Divide total force by pinned contact area underneath stage to get pressure Use Laplace pressure to get droplet curvature, assumed uniform across surface Calculate base contact angle from Lippmann-Young equation

Convert base contact angle to slope: dydx_0 = tan (CA_b-90); while loop

Loop through possible base radii starting with a lower bound guess: for base radius FROM lower_bound TO upper_bound BY step_size;

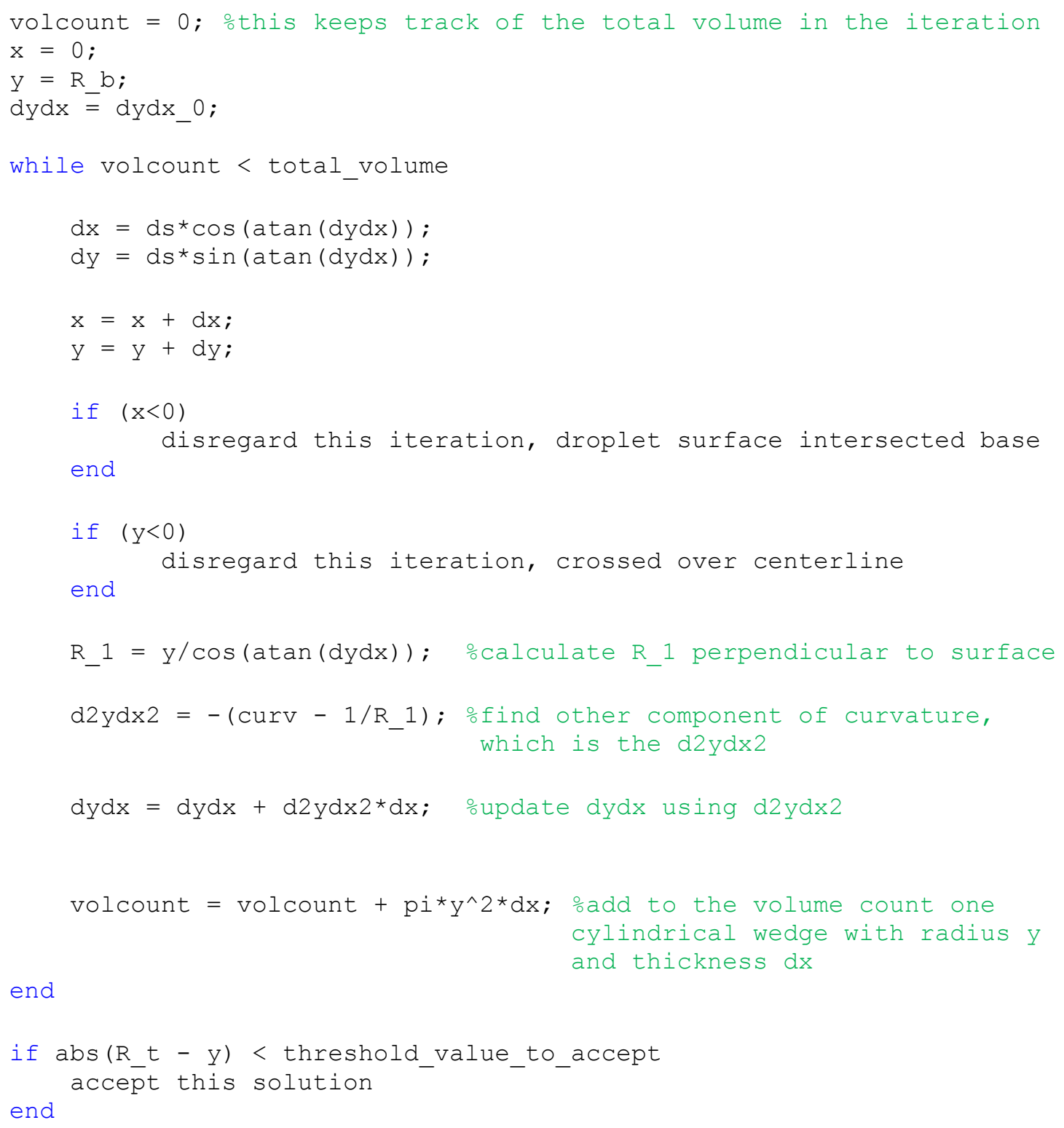

end for loop 


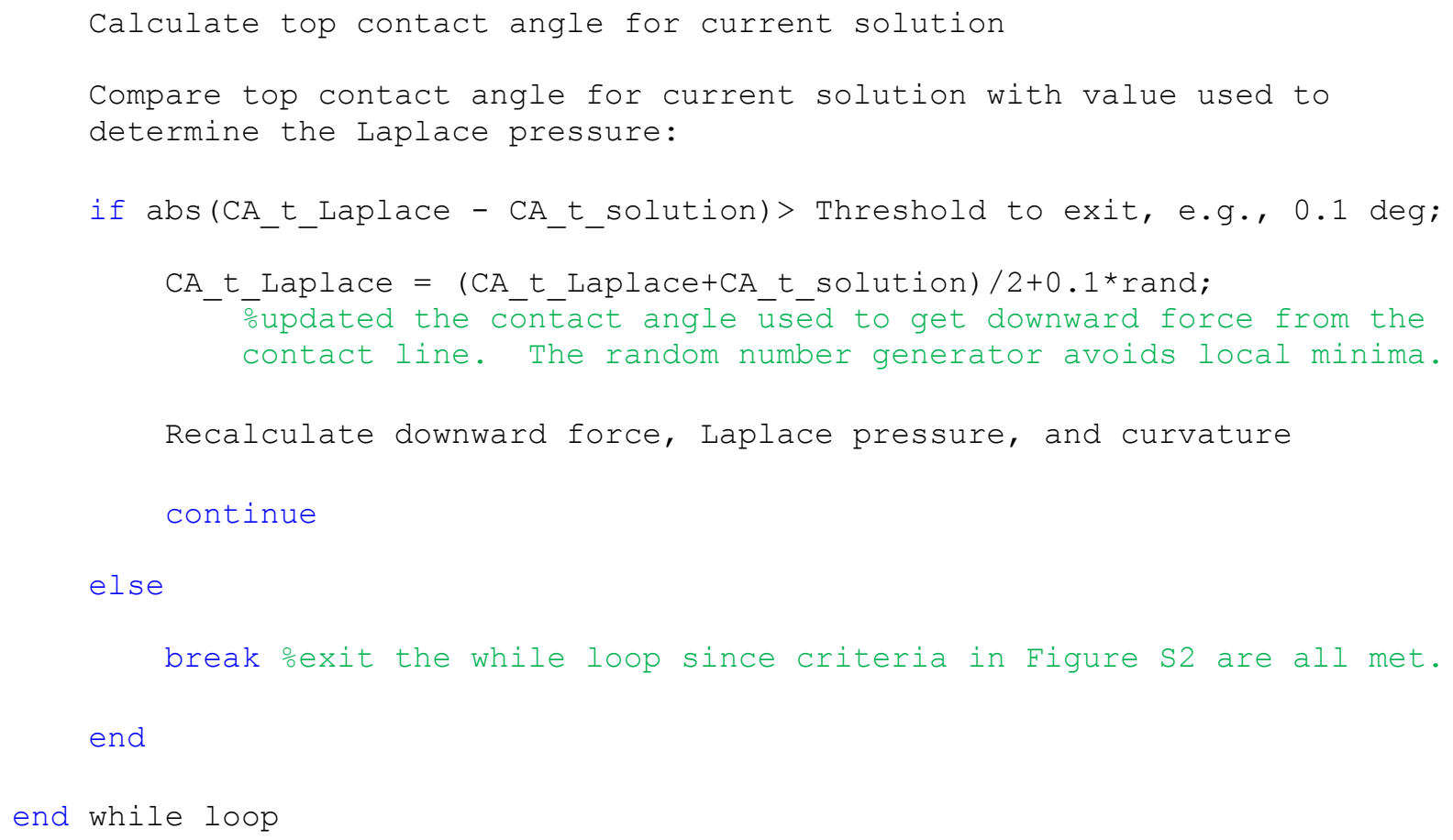

\section{Supplementary Information References}

T. Young, Phil. Trans. R. Soc. Lond. 95, 65 (1805).

2

C. Quilliet and B. Berge, Curr Opin Colloid In 6 (1), 34 (2001). 